\title{
Evaluation of Biochemical Markers in Multisystem Inflammatory Syndrome in Children (MIS-C) admitted in a Tertiary care hospital in Western Himalayan Region
}

\author{
Dr. Pancham Kumar ${ }^{1}$, Dr. Ambika ${ }^{2 *}$, Dr. Rakesh Sharma ${ }^{3}$, Dr. Kiran Azad ${ }^{4}$,Dr. Deepak Sharma \\ ${ }^{1}$ Associate Prof, Department of Pediatrics, Indira Gandhi Medical College, Shimla \\ ${ }^{2}$ Senior Resident, Department of Pediatrics, Indira Gandhi Medical College, Shimla \\ ${ }^{3}$ Prof \& Head, Department of Pediatrics, Indira Gandhi Medical College, Shimla \\ ${ }^{4}$ Dept. of Pediatrics, Indira Gandhi Medical College, Shimla \\ ${ }^{5} \mathrm{MMU}$, Solan
}

DOI: 10.29322/IJSRP.11.12.2021.p12049

http://dx.doi.org/10.29322/IJSRP.11.12.2021.p12049

\begin{abstract}
Background: The present study aimed to describe the Biochemical investigation among children admitted as a case Multisystem Inflammatory Syndrome in Children (MIS-C) in Indira Gandhi Medical college, Shimla.

Material \& Methods: We conducted a cross sectional study for MIS-C from January to July 2021, in the pediatric ward of Indira Gandhi Medical College Shimla in Himachal Pradesh. Children admitted with diagnosis of MISC were included in the study. Data regarding Biochemical investigation was extracted and analyzed using Epi Info V7 software.
\end{abstract}

Results: In the present study, a total of 31 children diagnosed and admitted as multisystem inflammatory syndrome in children (MIS-C) were included. 8(25.8\%) had a Covid antibody titre between 6-10. 23(74.2\%) had more than $10 \mathrm{gm} \%$ of $\mathrm{Hb}, 18(58.1 \%)$ had TLC increased for age,26(83.9\%) had neutrophils high for age, 30(96.8\%) had RBS in normal range, 23(80.6\%) had normal lipid profile, $15(48.4 \%)$ had platelets in range of 1-1.5lac, 14(45.2\%) had high PT , 18 (58.1\%) had normal range of INR, $17(54.8 \%)$ had normal range for APPT, 25(80.6\%) had normal range of B. Urea, $15(48.4 \%)$ had S creatinine levels twice for the upper limit, 12(38.7\%) had acidosis, 10(32.3\%) had azotemia,28(90.3\%) had normal range of Potassium (K+),28 (90.3\%) had normal range of sodium(Na+),10(32.3\%) D-DIMER twice the normal limit, $3(9.7 \%)$ had high within twice for the upper limit of TROP-1, 6(19.4\%) had CRP between 25-30, 12 (38.7\%) had ANC high for age, 9(29.0\%) had ESR more than four times, $9(29.0 \%)$ had ferritin high between twice to thrice for the upper limit and 10(32.3\%) had LDH patients had high between twice to thrice for the upper limit.

Conclusion: Most of the patients of MIS-C had raised inflammatory markers and deranged biochemical profile. Delay in biochemical investigation cause delay in diagnosis \& further referral may have adversely affected the prognosis as well as outcome

Index Terms- Biochemical investigation, Multisystem Inflammatory Syndrome in Children (MIS-C)

\section{INTRODUCTION}

$\mathrm{C}$ COVID-19, a severe viral respiratory infection caused by SARS-CoV- 2, affects almost every age group. Recently, a severe multi-system inflammatory syndrome (MIS-C) has been reported in individuals less than 21 years of age. ${ }^{1,2}$

MIS-C is diagnosed based on the symptoms (persistent fever and dysfunction of one or multiple organs, such as the heart or gastrointestinal system, etc), together with laboratory tests to look for markers of inflammation, as this, can seriously affect the organ systems like heart, blood vessels, kidneys, digestive system, brain, skin, etc. ${ }^{3,4}$

Children with MIS-C, present with fever as a major symptom and inflammation of various organ systems, which is confirmed by a multitude of laboratory tests. There are a number of varied diagnostic tests used by the medical professionals, to look for abnormal level of inflammatory markers. Once MIS-C is diagnosed, children need to be followed up over a period of time with laboratory investigations to assess inflammatory markers, coagulation profile, liver function, cardiac function, and other aspects of this illness. ${ }^{5,6}$

Children diagnosed with MIS-C require hospitalization and strict observation. Few of them also need admission to intensive care unit (ICU) .,7

There is a paucity of data regarding Biochemical investigation of MIS-C in this hilly region. Against this backdrop, the study was conducted to describe Biochemical investigation among children admitted as a case Multisystem Inflammatory Syndrome in Children (MIS-C) in Indira Gandhi Medical college, Shimla.

\section{AIMS \& OBJECTIVES}

To evaluate the Biochemical investigation associated with multisystem inflammatory syndrome in children (MIS-C). 


\section{MATERIAL \& METHODS}

Ours was a descriptive, cross sectional institutional based study, carried out in the Department of Pediatrics, Indira Gandhi Medical College, Shimla, Himachal Pradesh, located at an altitude of $2,276 \mathrm{~m}$. The study was conducted between January 2021 to July 2021. All children admitted to the pediatric ward in this time period with a proven diagnosis of MIS C, were included in the study. Approval was obtained from the concerned authorities of Indira Gandhi Medical College Shimla in Himachal Pradesh

\section{Operational definition for a case of MIS-C ${ }^{1}$}

Children and adolescents $0-19$ years of age with fever $>3$ days AND two of the following:

a) Rash or bilateral non-purulent conjunctivitis or mucocutaneous inflammation signs (oral, hands or feet).

b) Hypotension or shock.

c) Features of myocardial dysfunction, pericarditis, valvulitis, or coronary abnormalities (including ECHO findings or elevated Troponin/NT-proBNP),

d) Evidence of coagulopathy (by PT, PTT, elevated d-Dimers).

e) Acute gastrointestinal problems (diarrhoea, vomiting, or abdominal pain).

AND

Elevated markers of inflammation such as ESR, C-reactive protein, or procalcitonin.

\section{AND}

No other obvious microbial cause of inflammation, including bacterial sepsis, staphylococcal or streptococcal shock syndromes. AND

Evidence of COVID-19 (RT-PCR, antigen test or serology positive), or likely contact with patients with COVID-19.
The various biochemical tests were done in a standardized laboratory under supervision of a qualified medical personnel. Covid-19 serology was done by testing for the IgG antibodies against the anti-spike protein, using the $\mathrm{Cbc}$ was done by the automated cell counter, and random blood sugar and other biochemical tests were done by biochemical autoanalyzer. The test results were then extrapolated to standard reference ranges for pediatric age group.

\section{Data analysis}

The data were collected from the record files of admitted children, compiled and entered in MS Excel, and analyzed using appropriate statistical tools in software Epi info V7 by applying appropriate statistical test in terms of frequencies and percentage.

\section{RESULTS}

In the present study a total of 31 children were diagnosed and admitted as a case of multisystem inflammatory syndrome in children (MIS-C) in the pediatric ward of Indira Gandhi Medical College Shimla in Himachal Pradesh between Jan 2021- July 2021.

In our study, mean age of the children diagnosed as multisystem inflammatory syndrome (MIS-C) was 7.12 \pm 4.78 years. Maximum 12 children $(38.7 \%)$ were of age group 5-10 years followed by $10(32.3 \%)$ of age group 6-10 years , $8(25.8 \%)$ of $11-15$ years and $1(3.2 \%)$ of $15-19$ years age group. Out of the total of 31,16 children $(51.6 \%)$ were males while $15(48.4 \%)$ were females. $29(93.5 \%)$ belonged to rural area while 2(6.5\%) were from Urban area.(Figure-1)

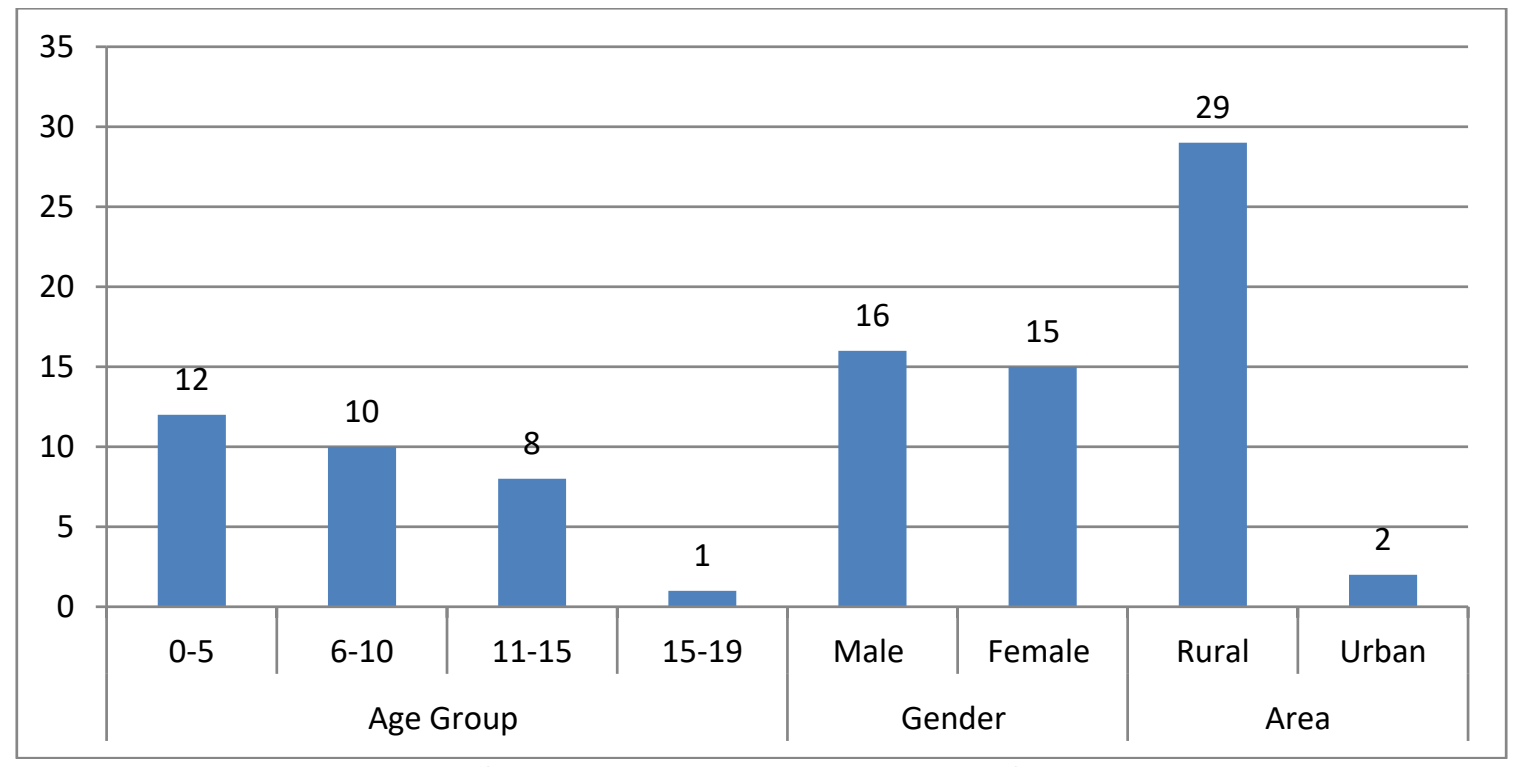

Figure -1: Socio-Demographic variables of MISC-C patients

All admitted patients of MIS-C were tested for Covid-19 at admission and were RAT \& RT-PCR negative. All children were sampled for Covid Serology, of which a maximum of $8(25.8 \%)$ had an antibody titre between 6-10, followed by, between 1-5 in
$6(19.4 \%)$ children, each between $11-15$ in $5(16.1 \%)$ patients, between $0-1 \& 16-20$ in $2(6.5 \%)$ patients each and between 21 $25 \& 46-50$ in 1 (3.2\%)patient each.(Table-2) 


\begin{tabular}{|l|l|l|l|}
\hline \multicolumn{2}{|c|}{} & Frequency & Percent \\
\hline RAT Results & Negative & 31 & 100.0 \\
\hline RT-PCR & Negative & 31 & 100.0 \\
\hline \multirow{4}{*}{$\begin{array}{l}\text { Covid-19Antibody } \\
\text { (IgG anti-spike) }\end{array}$} & $0-1$ & 2 & 6.5 \\
\cline { 2 - 4 } & $1-5$ & 6 & 19.4 \\
\cline { 2 - 4 } & $6-10$ & 8 & 25.8 \\
\cline { 2 - 4 } & $11-15$ & 5 & 16.1 \\
\cline { 2 - 4 } & $16-20$ & 2 & 6.5 \\
\cline { 2 - 4 } & $21-25$ & 1 & 3.2 \\
\cline { 2 - 4 } & $46-50$ & 1 & 3.2 \\
\cline { 2 - 4 } & $>100$ & 6 & 19.4 \\
\hline
\end{tabular}

Table-2: RAT \& RT-PCR Positivity and Antibody Titre among MIS-C patients

Among them 1(3.2\%) child had Hemoglobin ( $\mathrm{Hb})$ levels between $6-8 \mathrm{gm} \%, 7(22.6 \%)$ in the range of $8-10 \mathrm{gm} \%$, and $23(74.2 \%)$ more than $10 \mathrm{gm} \%$ of $\mathrm{Hb}$. In this study, $4(12.9 \%)$ children had a Total Leucocyte Count (TLC) of less than 5000/cmm, $9(29.0 \%)$ had TLC normal for age and 18 (58.1\%) had a TLC increased for age . Among the total, 2(6.5\%) children had an Absolute Neutrophilic Count (ANC) low for age, 3 (9.7\%) had normal ANC for age and 26(83.9\%) had high ANC for age. All children were tested for random blood sugar (RBS), by lancet method at admission, it was found that $30(96.8 \%)$ had RBS in the normal range for age, while only $1(3.2 \%)$ had low RBS. $23(80.6 \%)$ children had normal lipid profile while $6(19.4 \%)$ had deranged lipid profile, with hypertriglyceridemia. Among the total 31 children, $8(25.8 \%)$ had Platelet count, $15(48.4 \%)$ had platelets in the range of 1-1.5lac, 6(19.4\%) had thrombocytopenia in the range of $75 \mathrm{k}-1 \mathrm{lac}$ and $2(6.5 \%)$ severe thrombocytopenia in the range of 25-50 thousand.

Out of the 31children, 11(35.5\%) had Prothrombin time(PT) in normal range, $14(45.2 \%)$ had abnormal values of PT, twice the normal range ,4(12.9\%) had three times the upper limit,1(3.2\%) had more than four times and $1(3.2 \%)$ had PT more than five times of the normal range. Among the total of 31 patients, 18 $(58.1 \%)$ had INR in the normal range, $10(32.3 \%)$ had INR between $1-2,2(6.5 \%)$ had range for $2-3$ and $1(3.2 \%)$ had range of 3-4 of INR. Among them 17(54.8\%) had normal range APPT, $13(41.9 \%)$ had APPT between 1-2 range and 1(3.2\%) child had APPT between 2-3.(Table-3)

\begin{tabular}{|c|c|c|c|}
\hline & & Frequency & Percent \\
\hline \multirow{3}{*}{ HB } & $6-8 \mathrm{gm} \%$ & 1 & 3.2 \\
\hline & $8-10 \mathrm{gm} \%$ & 7 & 22.6 \\
\hline & $>10 \mathrm{gm} \%$ & 23 & 74.2 \\
\hline \multirow{3}{*}{ TLC } & $<5000 / \mathrm{cmm}$ & 4 & 12.9 \\
\hline & Normal for age & 9 & 29.0 \\
\hline & Increased for age & 18 & 58.1 \\
\hline \multirow{3}{*}{\begin{tabular}{|l} 
ABSOLUTE \\
NEUTROPHIL COUNT
\end{tabular}} & Low for age & 2 & 6.5 \\
\hline & Normal for age & 3 & 9.7 \\
\hline & High for age & 26 & 83.9 \\
\hline \multirow{2}{*}{ RBS } & Normal & 30 & 96.8 \\
\hline & Low & 1 & 3.2 \\
\hline \multirow{2}{*}{ Lipid profile } & Normal & 25 & 80.6 \\
\hline & High & 6 & 19.4 \\
\hline \multirow{3}{*}{ Platelets } & Normal & 8 & 25.8 \\
\hline & $1-1.5$ lacs & 15 & 48.4 \\
\hline & 75k-1lac & 6 & 19.4 \\
\hline
\end{tabular}




\begin{tabular}{|c|c|c|c|}
\hline & $25-50 \mathrm{k}$ & 2 & 6.5 \\
\hline \multirow{5}{*}{$\begin{array}{l}\text { Prothrombin time } \\
\text { PT }\end{array}$} & Normal & 11 & 35.5 \\
\hline & More than twice for the upper limit & 14 & 45.2 \\
\hline & More than three the upper limit & 4 & 12.9 \\
\hline & More than four times & 1 & 3.2 \\
\hline & More than five times & 1 & 3.2 \\
\hline \multirow{4}{*}{ INR } & Normal & 18 & 58.1 \\
\hline & 1 to 2 & 10 & 32.3 \\
\hline & 2 to 3 & 2 & 6.5 \\
\hline & 3 to 4 & 1 & 3.2 \\
\hline \multirow{3}{*}{ APPT } & Normal & 17 & 54.8 \\
\hline & 1 to 2 & 13 & 41.9 \\
\hline & 2 to 3 & 1 & 3.2 \\
\hline
\end{tabular}

Table-3: CBC, Blood sugar, Lipid and Coagulation profile

In this study, 25(80.6\%) children had normal range of B. Urea, and $6(19.4 \%)$ had high, twice the upper limit. Of the total, $9(29.0 \%)$ children, had normal range of $\mathrm{S}$ creatinine, $15(48.4 \%)$ had twice the upper limit, $5(16.1 \%)$ had between twice to thrice the upper limit and 2(6.5\%) had S creatinine more than four times the normal range of age. In this study, among the total 31,
12(38.7\%) had acidosis at presentation, and 10(32.3\%) patients had azotemia.

We also evaluated the serum electrolytes and of the 31 children, 28(90.3\%) had normal range of Potassium (K+), $2(6.5 \%)$ had hypokalemia and 1(3.25) hyperkalemia. Also, 28 (90.3\%)participants had normal range of sodium(Na+), 2(6.5\%) hyponatremia and $1(3.2 \%)$ child had hypernatremia.(Table-4)

\begin{tabular}{|c|c|c|c|}
\hline & & Frequency & Percent \\
\hline \multirow{2}{*}{ UREA } & Normal & 25 & 80.6 \\
\hline & High within twice for the upper limit & 6 & 19.4 \\
\hline \multirow{4}{*}{ S. Creatinine } & Normal & 9 & 29.0 \\
\hline & High within twice for the upper limit & 15 & 48.4 \\
\hline & High between twice to thrice the upper limit & 5 & 16.1 \\
\hline & More than four times & 2 & 6.5 \\
\hline \multirow{2}{*}{ ACIDOSIS } & Yes & 12 & 38.7 \\
\hline & No & 19 & 61.3 \\
\hline \multirow{2}{*}{ AZOTEMIA } & Yes & 10 & 32.3 \\
\hline & No & 21 & 67.7 \\
\hline \multirow{3}{*}{$\mathbf{K}+$} & Normal & 28 & 90.3 \\
\hline & Hpokalemia & 2 & 6.5 \\
\hline & Hyperkalemia & 1 & 3.2 \\
\hline \multirow{3}{*}{$\mathrm{Na}+$} & Normal & 28 & 90.3 \\
\hline & Hyponatremia & 2 & 6.5 \\
\hline & Hypernatremia & 1 & 3.2 \\
\hline
\end{tabular}

Table-4: Kidney Function and Electrolytes

Among the total 31 patients, 5(16.1\%) had D-DIMER in the normal range at admission, 10(32. 3\%) had two times the normal limit, $9(29.0 \%)$ had three times for the upper range, $4(12.9 \%)$ four times, $2(6.5 \%)$ had more than five times and $1(3.2 \%)$ patient had more than six times the normal limit of D-DIMER.
To study the impact on the Cardiovascular System, Troponin I testing was done, 4 (12.9\%) children had normal range of TROPI, $3(9.7 \%)$ had elevated levels twice the upper limit,1(3.2\%) had levels more than 5 times, $2(6.5 \%)$ patients had more than ten times while TROP-Iwas not performed for 21 patients. 
Among the total 31 patients, 1(3.2\%) had CRPlevels between 5-10, $3(9.7 \%)$ between 10-25, 6(19.4\%) between 25-30 , $5(16.1 \%)$ between $50-75,1(3.2 \%)$ patient had between 75 $100,1(3.2 \%)$ patient had between 100-150, 4(12.9\%) between
$150-200,4(12.9 \%)$ patients had between $200-250,2(6.5 \%)$ patients had between $250-300,3(9.7 \%)$ patients had between $300-350$ and $1(3.2 \%)$ patient had CRP increased more than 350.(Table-5)

\begin{tabular}{|l|l|l|l|}
\hline & & Frequency & \% \\
\hline \multirow{5}{*}{ D-DIMER } & Normal & 5 & 16.1 \\
\cline { 2 - 4 } & High within twice for the upper limit & 10 & 32.3 \\
\cline { 2 - 4 } & High between thrice for the upper limit & 9 & 29.0 \\
\cline { 2 - 4 } & More than four times & 4 & 12.9 \\
\cline { 2 - 4 } & More than five times & 2 & 6.5 \\
\cline { 2 - 4 } & More than six times & 1 & 3.2 \\
\hline \multirow{5}{*}{ TROP-I } & Not done & 21 & 67.7 \\
\cline { 2 - 4 } & Normal & 4 & 12.9 \\
\cline { 2 - 4 } & High within twice for the upper limit & 3 & 9.7 \\
\cline { 2 - 4 } & More than five times & 1 & 3.2 \\
\cline { 2 - 4 } & More than ten times & 2 & 6.5 \\
\hline & $5-10$ & 1 & 3.2 \\
\cline { 2 - 4 } & $10-25$ & 3 & 9.7 \\
\cline { 2 - 4 } & $25-50$ & 6 & 19.4 \\
\cline { 2 - 4 } & $50-75$ & 5 & 16.1 \\
\cline { 2 - 4 } & $75-100$ & 1 & 3.2 \\
\cline { 2 - 4 } & $100-150$ & 1 & 3.2 \\
\cline { 2 - 4 } & $150-200$ & 4 & 12.9 \\
\cline { 2 - 4 } & $200-250$ & 4 & 12.9 \\
\cline { 2 - 4 } & $250-300$ & 2 & 6.5 \\
\cline { 2 - 4 } & $300-350$ & 3 & 3.2 \\
\cline { 2 - 4 } & $>350$ & 1 & \\
\hline
\end{tabular}

Table-5: Trend of Inflammatory markers in MIS C children

Among the total 31 patients, $8(25.8 \%)$ had low Absolute Lympocyte Count (ALC) for age,11 (35.5\%) normal ALC for age and $12(38.7 \%)$ had ALC high for age. In this study among the total 31 patients, $3(9.7 \%)$ children had normal ESR, 4(12.9\%) had high within twice the upper limit, $8(25.8 \%)$ between twice to thrice the upper limit, $9(29.0 \%)$ more than four times, $5(16.1 \%)$ had more than five times, $1(3.2 \%)$ patient had more than seven times and $1(3.2 \%)$ had more than ten times the normal range of ESR. In our study, of the total of 31children, 3(9.7\%) had normal values of ferritin, $5(16.1 \%)$ had high ferritin levels within twice for the upper limit, $9(29.0 \%)$ had between two to three times the upper limit, 4(12.9\%) had more than four times, $2(6.5 \%)$ patients had more than five times, $3(9.7 \%)$ had more than six times , $1(3.2 \%)$ more than seven times, $1(3.2 \%)$ more than eight times and $3(9.7 \%)$ patients had more than ten times the normal value. Measurement of LDH levels, 3(9.7\%) had normal range,4(12.9\%) patients had high LDH levels within twice for the upper limit, $10(32.3 \%)$ children between twice to thrice for the upper limit, $7(22.6 \%)$ had more than four times, $6(19.4 \%)$ had more than five times and $1(3.2 \%)$ child very high LDH levels more than six times. (Table-6)

\begin{tabular}{|l|l|l|l|}
\hline \multicolumn{2}{|l|}{} & Frequency & Percent \\
\hline \multirow{4}{*}{ ALC } & Low for age & 8 & 25.8 \\
\cline { 2 - 4 } & Normal for age & 11 & 35.5 \\
\cline { 2 - 4 } & High for age & 12 & 38.7 \\
\hline \multirow{5}{*}{ ESR } & Normal ESR & 3 & 9.7 \\
\cline { 2 - 4 } & High within twice for the upper limit & 4 & 12.9 \\
\cline { 2 - 4 } & $\begin{array}{l}\text { High between two to three times the upper } \\
\text { limit }\end{array}$ & 8 & 25.8 \\
\cline { 2 - 4 } & More than four times & 9 & 29.0 \\
\cline { 2 - 4 } & More than five times & 5 & 3.2 \\
\cline { 2 - 4 } & More than seven times & 1 & 3.2 \\
\cline { 2 - 4 } & More than ten times & 1 & 9.7 \\
\hline \multirow{2}{*}{ FERRITIN } & Normal & 3 & 16.1 \\
\cline { 2 - 4 } & High within twice the upper limit & 5 & \\
\hline
\end{tabular}




\begin{tabular}{|l|l|l|l|}
\hline \multirow{5}{*}{} & High between two to three for the upper limit & 9 & 29.0 \\
\cline { 2 - 4 } & More than four times & 4 & 12.9 \\
\cline { 2 - 4 } & More than five times & 2 & 6.5 \\
\cline { 2 - 4 } & More than six times & 3 & 9.7 \\
\cline { 2 - 4 } & More than seven times & 1 & 3.2 \\
\cline { 2 - 4 } & More than eight times & 1 & 3.2 \\
\cline { 2 - 4 } & More than Ten times & 3 & 9.7 \\
\hline \multirow{5}{*}{ LDH } & Normal & 3 & 9.7 \\
\cline { 2 - 4 } & High within twice for the upper limit & 4 & 12.9 \\
\cline { 2 - 4 } & High between twice thrice for the upper limit & 10 & 32.3 \\
\cline { 2 - 4 } & More than four times & 7 & 22.6 \\
\cline { 2 - 4 } & More than five times & 6 & 19.4 \\
\cline { 2 - 4 } & More than six times & 1 & 3.2 \\
\hline
\end{tabular}

Table-6: Inflammatory markers in MIS C children

\section{Discussion}

In the present study, mean age of the children diagnosed as multisystem inflammatory syndrome in children (MIS-C) was $7.12 \pm 4.78$ years. Majority, $12(38.7 \%)$ were in the age group of 510 years followed by $10(32.3 \%)$ of age group 6-10 years , 8 (25.8\%) $11-15$ years and $1(3.2 \%)$ in $15-19$ years age group. $16(51.6 \%)$ out of 31 children were males while $15(48.4 \%)$ were females.29(93.5\%) belonged to rural area while 2(6.5\%) to Urban area

All of the 31 admitted MIS-C children were RAT \& RT-PCR negative. It means that children with MIS-C, test negative for a current infection with the COVID-19 virus. Covid serology results show that $8(25.8 \%)$ had antibody titre between 6-10. It indicates that many of these children were infected with the COVID19 virus in the recent past, as shown by positive IgG anti-spike antibody test results. An antibody test with a positive result means that the child's immune system developed antibodies, following a past infection with COVID-19 virus. Sometimes, the Covid Serology positivity is the only indication that the child was ever infected with Covid-19, as there may not be any history of Covid19 infection in the child or close contacts, meaning that the child may have contracted the virus and remained asymptomatic. ${ }^{5}$

In the present study, $23(74.2 \%)$ had more than $10 \mathrm{gm} \%$ of $\mathrm{Hb}, 18(58.1 \%)$ had TLC increased for age ,26(83.9\%) had neutrophils high for age, 30(96.8\%) had RBS in normal range, $23(80.6 \%)$ had normal lipid profile, $15(48.4 \%)$ had platelets in range of 1-1.5lac, 14(45.2\%) had high PT within twice for the limit , $18(58.1 \%)$ had normal range of INR, 17(54.8\%) had normal range for APPT, 25(80.6\%) had normal range of B. Urea, $15(48.4 \%)$ had S creatinine high within twice for the upper limit, $12(38.7 \%)$ had acidosis, $10(32.3 \%)$ had azotemia,28(90.3\%) had normal range of Potassium $(\mathrm{K}+), 28(90.3 \%)$ had normal range of sodium(Na+),10(32.3\%) had D-DIMER twice the normal limit, 3 (9.7\%) had high within twice for the upper limit of TROP-I, 6(19.4\%) had CRP between 25-30, 12 (38.7\%) had ALC high for age, 9(29.0\%) had ESR more than four times, 9(29.0\%) had ferritin high between twice to thrice for the upper limit and $10(32.3 \%)$ had LDH patients had high between twice to thrice for the upper limit.

Similar types of results were observed in the studies done by Levi Hoste et $\mathrm{al}^{8}$, Fouriki A et $\mathrm{al}^{9}$, Leora $\mathrm{R}$ et $\mathrm{al}^{10}{ }^{10}, \mathrm{M}$. Ahmed et $\mathrm{al}^{11}$, Gupta $\mathrm{S}$ et $\mathrm{al}^{12} \&$ Tolunay $\mathrm{O}$ et $\mathrm{al}^{13}$. These studies also showed raised inflammatory markers ,electrolyte abnormalities, increased markers of Disseminated intravascular coagulation (DIC) such as D-dimer and/or deranged coagulation profile ,raised Serum lactate dehydrogenase (LDH) and tissue markers neutrophilia, increased CRP, ESR, ferritin, troponin but lower lymphocytes and platelets in MIS-C cases.

Usually MIS-C patients present with similar but also with different and unique characteristics. In case of delayed diagnosis and treatment, morbidity and mortality rates may increase. Therefore, the awareness and knowledge of all physicians, especially those are dealing with pediatric patients, about MIS-C should be increased. ${ }^{13}$

\section{CONCLUSION}

High index of suspicion about MIS-C especially in severe critical cases in the time of Covid-19 pandemic is the need of the hour. MIS-C may have varied clinical presentations in different geographical areas. Most of the patients of MIS-C had raised inflammatory markers and deranged biochemical profile. More detailed and extensive work up for inflammatory markers in MIS$\mathrm{C}$ cases presenting late with negative RTPCR are warranted to be able to understand and form guidelines regarding management of MIS-C cases. Delay in biochemical investigation cause delay in diagnosis \& further referral which may adversely affect the prognosis as well as outcome.

\section{Conflict of Interest: None}

\section{Funding: None}

\section{REFERENCES}

[1] World Health Organization (WHO). Available at https://www.who.int/news-room/commentaries/detail/multisysteminflammatory-syndrome-in-children-and-adolescents-with-covid-19 (Accessed on 23 July 2021)

[2] Up to Date. Available at: https://www.uptodate.com/contents/covid-19multisystem-inflammatory-syndrome-in-children-mis-c-clinical-featuresevaluation-and-diagnosis(Accessed on 24 July 2021) 
[3] Mayo Clinic. Available at: https://www.mayoclinic.org/diseasesconditions/mis-c-in-kids-covid-19/symptoms-causes/syc20502550(Accessed on 25 July 2021)

[4] Childrens Hospital. https://www.childrenshospital.org/conditions-andtreatments/conditions/m/mis-c(Accessed on 26 July 2021)

[5] Mayo Clinic. Available at: https://www.mayoclinic.org/diseasesconditions/mis-c-in-kids-covid-19/diagnosis-treatment/drc20502561(Accessed on 26 July 2021)

[6] Healthy Children. Available at: https://www.healthychildren.org/English/health-issues/conditions/COVID19/Pages/covid_inflammatory_condition.aspx(Accessed on 27 July 2021)

[7] Center for Disease Control \& Prevention .Available at: https://www.cdc.gov/mis/mis-c.html(Accessed on 28 July 2021)

[8] Levi Hoste, Ruben Van Paemel \& Filomeen Haerynck.Multisysteminflammatory syndrome in children related to COVID-19:a systematic review. European Journal of Pediatrics,2021 .https:// doi.org/10.1007/s00431-021-03993-5

[9] Fouriki A, Fougère Y, De Camaret C, Blanchard Rohner G, Grazioli S, Wagner N, Relly C, Pachlopnik Schmid J, Trück J, Kottanatu L, Perez E, Perez M-H, Schaffner D, Asner SA and Hofer M (2021) Case Report: Case Series of Children With Multisystem Inflammatory Syndrome Following SARS-CoV-2 Infection in Switzerland. Front. Pediatr. 8:594127.

[10] Leora R. Characteristics and Outcomes of US Children and Adolescents With Multisystem Inflammatory Syndrome in Children (MIS-C) Compared With Severe Acute COVID-19 JAMA. 2021;325(11):1074-1087.

[11] M. Ahmed et al. Multisystem inflammatory syndrome in children: A systematic review EClinicalMedicine. 26 (2020) 100527.
[12] Gupta S, Chopra N, Singh A, et al. Unusual Clinical Manifestations and Outcome of Multisystem Inflammatory Syndrome in Children (MIS-C) in a Tertiary Care Hospital of North India. J Trop Pediatr. 2021;67(1):fmaa127.

[13] Tolunay O, Çelik Ü, Arslan İ, et al. Multisystem Inflammatory Syndrome in Children (MIS-C) Associated with COVID-19: A Case Series Experience in a Tertiary Care Hospital of Southern Turkey. J Trop Pediatr. 2021;67(2):fmab050.

\section{AUTHORS}

First Author - Dr. Pancham Kumar, Associate Prof, Department of Pediatrics, Indira Gandhi Medical College, Shimla

Second Author - Dr. Ambika, Senior Resident, Department of Pediatrics, Indira Gandhi Medical College, Shimla

Third Author - Dr. Rakesh Sharma, Prof \& Head, Department of Pediatrics, Indira Gandhi Medical College, Shimla

Fourth Author - Dr. Kiran Azad, Dept. of Pediatrics, Indira Gandhi Medical College, Shimla

Fifth Author - Dr. Deepak Sharma, MMU, Solan

Corresponding Author: Dr. Ambika Sood

Email: drambikasood@gmail.com 